\title{
Diversidade de moscas-das-frutas, suas plantas hospedeiras e seus parasitóides nas regiões Norte e Noroeste do Estado do Rio de Janeiro, Brasil
}

\author{
Fruit fly diversity, their host plants and their parasitoids in the northern and northwestern regions \\ of Rio de Janeiro State, Brazil
}

\author{
Michela Rocha Leal ${ }^{\text {* }}$ Silvana Aparecida da Silva Souza' ${ }^{\text {II }}$ Elen de Lima Aguiar-Menezes ${ }^{\text {II }}$ \\ Mauri Lima Filho ${ }^{\mathrm{IV}}$ Eurípedes Barsanulfo Menezes ${ }^{\mathrm{V}}$
}

RESUMO

Em razão da exploração crescente da fruticultura nas regiões Norte e Noroeste do Estado do Rio de Janeiro e da importância econômica e quarentenária das moscas-das-frutas para essa atividade agrícola no mundo, este estudo foi conduzido em cinco municípios dessas regiões: Cambuci, Campos dos Goytacazes, Itaocara, São Francisco do Itabapoana e São João da Barra, com objetivo de registrar as espécies de moscas-das-frutas, suas plantas hospedeiras e seus parasitóides. Os espécimes foram coletados por meio de armadilhas McPhail com solução aquosa de proteína hidrolisada a 5\% e em amostras de frutos. Nas armadilhas, $4 \%$ do total de moscas-das-frutas capturadas foram Ceratitis capitata e 96\% eram do gênero Anastrepha, num total de 15 espécies. Das moscas capturadas nas armadilhas, apenas seis espécies infestaram frutos entre as 12 espécies de fruteiras amostradas. Fruteiras das famílias Anacardiaceae, Malpighiaceae, Myrtaceae, Oxalidaceae, Passifloraceae e Sapotaceae foram plantas hospedeiras de moscas-das-frutas. Apenas três espécies de Anastrepha (A. fraterculus, A. sororcula e A. zenildae) infestaram goiabas da variedade comercial Paluma. Os parasitóides de larvas de moscas-das-frutas encontrados foram Doryctobracon areolatus e Aganaspis pelleranoi, parasitando-as em frutos de goiabeira e de outras fruteiras nessas regiões.

Palavras-chave: Tephritidae, Braconidae, Figitidae, relação tri-trófica.

\section{ABSTRACT}

Due to the increase in fruit crops acreage in the northern and northwestern regions of Rio de Janeiro State and to the economic and quarantine importance of the fruit flies to this agricultural activity in the world, this study was carried out in five municipalities of these regions: Cambuci, Campos dos Goytacazes, Itaocara, São Francisco do Itabapoana, and São João da Barra, aiming to study the fruit fly species, their host plants and their parasitoids. The specimens were collected using McPhail traps with aqueous solution of hydrolyzed protein to $5 \%$ and in fruit samples. In the traps, $4 \%$ of the total of fruit flies captured were Ceratitis capitata and $96 \%$ were from the genus Anastrepha in a total of 15 species. From these flies captured in the traps, only six species infested fruits among the 12 species of fruits sampled. Fruit trees of the families Anacardiaceae, Malpighiaceae, Myrtaceae, Oxalidaceae, Passifloraceae and Sapotaceae were host plants of fruit flies. Only three species of Anastrepha (A. fraterculus, A. sororcula and A. zenildae) infested guavas of the commercial variety Paluma. The parasitoids of fruit fly larvae found were Doryctobracon areolatus and Aganaspis pelleranoi, parasitizing them in fruits of guava and other fruit trees in these regions.

Key words: Tephritidae, Braconidae, Figitidae, tri-trophic relationship.

\section{INTRODUÇÃO}

As espécies de moscas-das-frutas Anastrepha spp. Schiner e Ceratitis capitata Wiedemann (Diptera: Tephritidae) destacam-se como as de maior importância econômica para a fruticultura brasileira (ZUCCHI, 2007). Essa espécie é originária do

\footnotetext{
IPrograma de Pós-graduação em Fitossanidade e Biotecnologia Aplicada, Universidade Federal Rural do Rio de Janeiro (UFRRJ), Rodovia BR 465, Km 7, 23890-000, Seropédica, RJ, Brasil. E-mail: michaleal@yahoo.com.br. *Autor para correspondência.

IIPrograma de Pós-graduação em Entomologia, Universidade Federal de Viçosa (UFV), Viçosa, MG, Brasil.

IIIEmbrapa Agrobiologia, Laboratório de Controle Biológico, Seropédica, RJ, Brasil.

${ }^{\mathrm{VI} C}$ Campus Dr. Leonel Miranda, UFRRJ, Estrada do Açúcar, km 5, 28020-560, Campos dos Goytacazes, RJ, Brasil.

vepartamento de Entomologia e Fitopatologia, Instituto de Biologia, UFRRJ, Seropédica, RJ, Brasil.
} 
Mediterrâneo e é a única deste gênero que ocorre no Brasil, onde infesta frutos de 58 espécies botânicas (ZUCCHI, 2001). O gênero Anastrepha é nativo das Américas, sendo que das 212 espécies conhecidas 99 ocorrem no Brasil e 28 estão catalogadas para o Estado do Rio de Janeiro (ZUCCHI, 2007; AGUIAR-MENEZES \& MENEZES, 2000; AGUIAR-MENEZES et al., 2004; FERRARA et al., 2004; SOUZA et al., 2008). Todavia, apesar dos esforços, não são conhecidos os hospedeiros para $56 \%$ das espécies brasileiras desse gênero (ZUCCHI, 2007). Os registros mais atuais para o Estado do Rio de Janeiro mostram que as plantas hospedeiras de 12 espécies de Anastrepha ainda não são conhecidas (AGUIAR-MENEZES \& MENEZES, 2000; AGUIAR-MENEZES et al., 2004; SOUZA et al., 2005; SOUZA et al., 2007; SOUZA et al., 2008). Com relação aos parasitóides de moscas-das-frutas, levantamentos já foram realizados em alguns municípios da região Metropolitana e do Sul do Estado do Rio de Janeiro (AGUIAR-MENEZES \& MENEZES, 2000; AGUIAR-MENEZES et al., 2004; SOUZA et al., 2005; SOUZA et al., 2007).

As regiões Norte e Noroeste desse Estado e, mais recente, as regiões das Baixadas Litorâneas e Serrana vêm recebendo incentivo do governo estadual, por meio do programa setorial Frutificar, para a expansão da fruticultura irrigada, principalmente com as culturas da goiaba, do maracujá-azedo e do abacaxi (SEAAPI, 2008). Entretanto, essa expansão tende a aumentar os problemas fitossanitários, destacando-se aqueles causados por moscas-das-frutas, particularmente porque as larvas desses dípteros comumente infestam uma grande variedade de frutos, incluindo goiaba (ZUCCHI, 2007; GOULD \& RAGA, 2002; AGUIAR-MENEZES et al., 2004; RAGA et al., 2006). Nas regiões Norte e Noroeste fluminense, a goiabeira (P. guajava) é amplamente cultivada, principalmente para a indústria de processamento, sendo a variedade Paluma a mais comum nos pomares comerciais dessa região (BRANDÃO, 2004).

No Estado do Rio de Janeiro, ainda existem poucos dados de distribuição geográfica das moscasdas-frutas, suas plantas hospedeiras e seus parasitóides, os quais se concentram em alguns municípios da região Metropolitana (AGUIARMENEZES \& MENEZES, 2000; AGUIAR-MENEZES et al., 2004; SOUZA et al., 2007), sendo que, mais recentemente, há dados para um município da região Sul (SOUZA et al., 2005) e para outro das Baixadas Litorâneas (SOUZA et al., 2008). Existem alguns novos registros de ocorrência de moscas-das-frutas para alguns municípios da região Noroeste, mas sem relacionar suas plantas hospedeiras e parasitóides (FERRARA et al., 2004).
Em razão da exploração crescente da fruticultura nas regiões Norte e Noroeste do Estado do Rio de Janeiro e da importância econômica e quarentenária das moscas-das-frutas para essa atividade agrícola no mundo, este trabalho teve como objetivos investigar a ocorrência e a diversidade de moscas-das-frutas nessas regiões e aumentar o conhecimento sobre a distribuição desse grupo de insetos nesse Estado, suas plantas hospedeiras e seus parasitóides.

\section{MATERIAL E MÉTODOS}

Área e período de estudo

O estudo foi conduzido no período de maio de 2005 a abril de 2007, em pomares comerciais de goiaba (Psidium guajava L.) var. Paluma, e em pomares domésticos diversificados, abrangendo os municípios

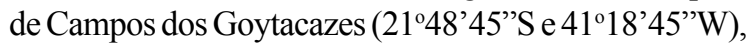
São Francisco do Itabapoana $\left(21^{\circ} 18^{\prime} 45^{\prime} \mathrm{S}\right.$ e $\left.40^{\circ} 56^{\prime} 15^{\prime \prime} \mathrm{W}\right)$ e São João da Barra (21\%41'15'S e $\left.41^{\circ} 03^{\prime} 45^{\prime \prime} \mathrm{W}\right)$, na região Norte do Estado do Rio de Janeiro, e o município de Itaocara $\left(21^{\circ} 41^{\prime} 15^{\prime}\right.$ 'S e $42^{\circ} 03$ ' $45^{\prime}$ 'W), na região Noroeste desse Estado. Ainda nesta região, o estudo foi conduzido no pomar experimental de goiaba (P. guajava) var. Paluma do Centro Agropecuário de Santo Antão (DFA-RJ/MAPA) no município de Cambuci (2133'45"S e 4156'15'W).

\section{Captura das moscas-das-frutas}

Os adultos de moscas-das-frutas foram capturados por meio de armadilhas do tipo frasco caçamoscas de plástico transparente, modelo McPhail, contendo $500 \mathrm{ml}$ de solução aquosa de proteína hidrolisada a 5\% (BioAnastrepha ${ }^{\circledR}$ ) como atrativo alimentar. Essa armadilha foi pendurada nas plantas, com auxílio de arame, a uma altura entre 1,5 a 2,0m do solo e numa densidade de uma armadilha por hectare. Os frascos foram inspecionados a cada sete ou 15 dias, quando os espécimes capturados eram coletados e o atrativo era substituído. Esses espécimes foram enxaguados com água numa peneira e foram acondicionados em frascos de vidro com álcool hidratado a $70 \%$, sendo devidamente etiquetados. No laboratório, os machos e as fêmeas dos gêneros Anastrepha e Ceratitis foram triados, sendo conservados em álcool hidratado a $70 \%$ para a posterior identificação da espécie.

Avaliação das plantas hospedeiras e parasitóides

Nas áreas amostradas com os frascos, com exceção de Cambuci, foram coletadas amostras de frutos de 16 espécies frutíferas, em seus respectivos períodos 
de amadurecimento, distribuídas entre sete famílias botânicas: Anacardiaceae [Spondias dulcis Forst. (cajámanga; abril-julho), Spondias lutea L. (cajá-mirim; março-maio) e Spondias purpurea L. (serigüela; fevereiro a abril)], Malpighiaceae [Malpighia glabra L. (acerola; março-maio)], Myrtaceae [Blepharocalyx salicifolius (Kunth) (cambuí; agosto-setembro), Eugenia uniflora L. (pitanga; outubro-janeiro), Eugenia tomentosa Camb. (cabeludinha; novembrodezembro), Psidium acutangulum DC. (araçá-pera; janeiro-março), Psidium cattleianum Sab. (araçá-decoroa; fevereiro-maio), Psidium guajava L. (goiaba comercial var. Paluma; safra: dezembro-março)], Oxalidaceae [Averrhoa carambola L. (carambola; março-maio e agosto-setembro)], Passifloraceae [Passiflora alata Dryand (maracujá-doce; abrilagosto), Passiflora sp. (maracujá-silvestre; abril-maio)], Rubiaceae [Coffea arabica L. (café; março a maio)] e Sapotaceae [Chrysophyllum cainito L. (abiu-roxo; outubro-novembro) e Pouteria torta (Mart.) (abiuamarelo; maio-julho)].

Os frutos das 16 espécies frutíferas colhidos no estado maduro, com consistência firme, na planta e no solo, foram transferidos para o laboratório, onde as amostras de cada espécie frutífera foram colocadas separadamente em bandejas plásticas de $37 \times 24 \times 7 \mathrm{~cm}$, sobre uma camada de $\pm 2 \mathrm{~cm}$ de areia peneirada, autoclavada e umedecida usada como substrato para as larvas frugívoras empuparem.

Em março/2006, 50 goiabas $(7,53 \mathrm{~kg}) \mathrm{da}$ variedade comercial Paluma, maduras e com consistência firme, foram coletadas, na planta e no solo, de um pomar comercial não tratado com inseticidas, em São Francisco do Itapaboana. Os frutos foram levados para o laboratório, pesados e acondicionados individualmente em potes plásticos transparentes de um litro com tampa telada de organza, sobre uma camada $\mathrm{de} \pm 4 \mathrm{~cm}$ de areia para as larvas frugívoras empuparem.

Periodicamente as bandejas e os potes foram examinados para verificar a presença de pupários. Os pupários encontrados foram contados e transferidos para copos plásticos transparentes de $250 \mathrm{ml}$, tendo-se o cuidado de envolvê-los em areia umedecida. Os copos foram acondicionados em potes plásticos de dois litros de capacidade e tampados com organza para promover a ventilação e evitar a fuga dos adultos emergidos. Esse material foi mantido em condições de temperatura e umidade ambiente $\left(25 \pm 3^{\circ} \mathrm{C}\right.$ e $80 \%$ UR) e foi inspecionado diariamente por um período de 30 dias após o início da formação dos pupários, para verificar a necessidade de umedecer a areia e observar a emergência das moscas e dos parasitóides. Adultos das moscas permaneceram nos potes alimentados com mel por um período de quatro dias após a emergência, para fixação da sua coloração e melhor esclerotização do ovipositor das fêmeas. A remoção desses insetos foi facilitada paralisando-os em congelador por cinco a 10 minutos. Posteriormente, foram mortos e conservados em álcool hidratado a $70 \%$ para posterior identificação específica.

\section{Identificação taxonômica}

Os adultos de moscas-das-frutas e de parasitóides foram separados por sexo e contados. Para a identificação das espécies, as fêmeas de Anastrepha foram examinadas sob microscópio estereoscópico e foram submetidas à identificação específica, com base no ovipositor, seguindo a metodologia descrita em ZUCCHI (2000). Como os machos de Anastrepha não podem ser identificados, por não apresentarem características morfológicas para o seu reconhecimento específico, estes foram identificados como Anastrepha spp. Exemplares de moscas do gênero Ceratitis foram identificados ao nível de espécie pelo diagnóstico das características morfológicas descritas por FOOTE (1980). Para o reconhecimento das espécies de parasitóides, os adultos foram examinados sob microscópio estereoscópico e foram submetidos à identificação específica, a partir de CANAL \& ZUCCHI (2000) e GUIMARÃES et al. (2000).

\section{Parâmetros avaliados}

A percentagem de parasitismo $(\% \mathrm{P})$ foi calculada de acordo com a seguinte equação: $\% \mathrm{P}=\mathrm{nx}$ $100 / \mathrm{N}$, em que $\mathrm{n}=$ número de parasitóides emergidos e $\mathrm{N}=$ número total de pupários. Os pupários sem emergência de adultos foram dissecados para verificar se a causa da mortalidade foi por parasitismo, de modo que o número de parasitóides não emergidos contidos nesses pupários foi considerado no cálculo da \% $\mathrm{P}$. A riqueza de espécies (S) de moscas-das-frutas foi igual ao número total de espécies capturadas em cada município. A freqüência relativa das espécies de moscas-das-frutas (F) foi obtida pela seguinte equação: $\mathrm{F}=\mathrm{n} \times 100 / \mathrm{N}$, em que $\mathrm{n}=$ número de adultos de uma determinada espécie e $\mathrm{N}=$ número total de adultos de moscas-das-frutas obtido por captura nas armadilhas McPhail ou coletados a partir de cada amostra de frutos. Foi estimado o índice médio de infestação das goiabas da variedade Paluma, sendo expresso de duas formas: a) número médio de pupários/fruto, o qual foi determinado pelo seguinte cálculo: número total de pupários obtidos da amostra de 50 goiabas dividido por esse total de frutos, e b) número médio de pupários/ $\mathrm{kg}$ de frutos, o qual foi determinado pelo seguinte cálculo: número total de pupários obtidos da amostra de 50 goiabas dividido pelo peso total dessa amostra. 


\section{RESULTADOS E DISCUSSÃO}

No período de 24 meses de levantamento, os frascos caça-moscas capturaram um total de 14672 espécimes de moscas-das-frutas (5383 machos e 9289 fêmeas), sendo que $96 \%$ e $4 \%$ pertenceram ao gênero Anastrepha e à Ceratitis capitata, respectivamente. No total, 16 espécies de moscas-das-frutas foram capturadas, sendo 15 espécies de Anastrepha e $\boldsymbol{C}$. capitata (Tabela 1). A ocorrência dessas espécies, no Estado do Rio de Janeiro, já fora registrada (AGUIARMENEZES \& MENEZES, 2000,AGUIAR-MENEZES et al., 2004; FERRARA et al., 2004; SOUZA et al., 2005; SOUZA et al., 2007; SOUZA et al., 2008). Entretanto, a riqueza de espécies diferiu entre os municípios, sendo a maior em São Francisco do Itabapoana $(S=15)$ e a menor em São João da Barra e Itaocoara $(\mathrm{S}=5)$, variando a espécie predominante (Tabela 1). A. obliqua ocorreu em maior freqüência em Campos dos Goytacazes, em relação aos demais tefritídeos capturados nesse município, $A$. fraterculus foi a espécie mais freqüente em São Francisco do Itabapoana, Cambuci e Itaocoara e A. sororcula, em São João da Barra (Tabela 1).

São Francisco de Itabapoana apresentou o maior número de espécimes de moscas-das-frutas $(61 \%$ do total capturado), o que pode ter sido devido a uma maior diversidade de plantas potencialmente hospedeiras. Das 10 espécies frutíferas amostradas nesse município, frutos de apenas três espécies não foram infestados por esses tefritídeos: cabeludinha (Eugenia tomentosa), maracujá-silvestre (Passiflora sp.) e café (Coffea arabica). Em São João da Barra, moscas-das-frutas também não infestaram frutos de cambuí (Blepharocalyx salicifolius) (Tabela 2).

Portanto, das 16 espécies frutíferas amostradas, 12 foram infestadas por moscas-das-frutas, obtendo-se de seus frutos um total de 1.118 espécimes (526 machos e 592 fêmeas) (Tabela 2). Anastrepha correspondeu a $99,5 \%$ desse total e os $0,5 \%$ restantes foram de C. capitata. Todavia, a proporção entre os indivíduos das diferentes espécies de tefritídeos variou com a espécie frutífera.

Apenas machos de Anastrepha foram obtidos das amostras de cajá-manga e araçá-pera (Tabela 2). Para as demais espécies botânicas, as espécies de moscas-das-frutas infestantes estão relacionadas na tabela 2 . Os resultados das identificações das moscas obtidas a partir das amostras de frutos culminaram em seis espécies de Anastrepha, além de C. capitata, sendo que exemplares dessas espécies também foram capturados nas armadilhas (Tabela 1).

Observou-se que três espécies de Anastrepha podem infestar um mesmo fruto da

Tabela 1 - Número $(\mathrm{N})$, freqüência relativa $(\mathrm{F})$ de fêmeas e riqueza de espécies $(\mathrm{S})$ de moscas-das-frutas (Diptera: Tephritidae) capturadas nas armadilhas McPhail, em três municípios da região Norte e dois municípios da região Noroeste do Estado do Rio de Janeiro (maio de 2005 a abril de 2007).

\begin{tabular}{|c|c|c|c|c|c|c|c|c|c|c|}
\hline \multirow{2}{*}{ Espécies } & \multicolumn{2}{|c|}{$\begin{array}{l}\text { Campos dos } \\
\text { Goytacazes }\end{array}$} & \multicolumn{2}{|c|}{$\begin{array}{l}\text { São Francisco do } \\
\text { Itabapoana }\end{array}$} & \multicolumn{2}{|c|}{ São João da Barra } & \multicolumn{2}{|c|}{ Cambuci } & \multicolumn{2}{|c|}{ Itaocara } \\
\hline & $\mathrm{N}$ & $\mathrm{F}$ & $\mathrm{N}$ & $\mathrm{F}$ & $\mathrm{N}$ & $\mathrm{F}$ & $\mathrm{N}$ & $\mathrm{F}$ & $\mathrm{N}$ & $\mathrm{F}$ \\
\hline Anastrepha barbiellinii Lima & 0 & 0 & 2 & 0,04 & 0 & 0 & 0 & 0 & 0 & 0 \\
\hline A. consobrina (Loew) & 0 & 0 & 4 & 0,1 & 0 & 0 & 0 & 0 & 0 & 0 \\
\hline A. distincta Greene & 2 & 0,1 & 7 & 0,1 & 2 & 0,4 & 8 & 1,6 & 2 & 0,2 \\
\hline A. fraterculus (Wiedemann) & 437 & 26,2 & 3165 & 56,3 & 66 & 12,5 & 338 & 68,1 & 750 & 77,0 \\
\hline A. grandis (Macquart) & 1 & 0,1 & 0 & 0 & 0 & 0 & 0 & 0 & 1 & 0,1 \\
\hline A. lutzi Lima & 0 & 0 & 1 & 0,02 & 0 & 0 & 0 & 0 & 0 & 0 \\
\hline A. manihoti Lima & 0 & 0 & 22 & 0,4 & 0 & 0 & 0 & 0 & 0 & 0 \\
\hline A. minensis Lima & 0 & 0 & 2 & 0,04 & 0 & 0 & 0 & 0 & 0 & 0 \\
\hline A. montei Lima & 0 & 0 & 185 & 3,3 & 0 & 0 & 0 & 0 & 0 & 0 \\
\hline A. obliqua (Macquart) & 770 & 46,2 & 1288 & 22,9 & 6 & 1,1 & 47 & 9,5 & 63 & 6,5 \\
\hline A. pickeli Lima & 0 & 0 & 13 & 0,2 & 0 & 0 & 0 & 0 & 0 & 0 \\
\hline A. pseudoparallela (Loew) & 10 & 0,6 & 3 & 0,1 & 0 & 0 & 1 & 0,2 & 0 & 0 \\
\hline A. serpentina (Wiedemann) & 1 & 0,1 & 124 & 2,2 & 0 & 0 & 0 & 0 & 0 & 0 \\
\hline A. sororcula Zucchi & 108 & 6,5 & 728 & 12,9 & 383 & 72,3 & 77 & 15,5 & 158 & 16,2 \\
\hline A. zenildae Zucchi & 0 & 0 & 18 & 0,3 & 0 & 0 & 0 & 0 & 0 & 0 \\
\hline Ceratitis capitata (Wied.) & 338 & 20,3 & 60 & 1,1 & 73 & 13,8 & 25 & 5,0 & 0 & 0 \\
\hline Total & 1667 & 100 & 5622 & 100 & 530 & 100 & 496 & 100 & 974 & 100 \\
\hline Riqueza de espécies (S) & \multicolumn{2}{|c|}{8} & \multicolumn{2}{|c|}{15} & \multicolumn{2}{|c|}{5} & \multicolumn{2}{|c|}{6} & \multicolumn{2}{|c|}{5} \\
\hline
\end{tabular}


Tabela 2 - Número de pupários (TP), número de machos de moscas (M), número de fêmeas de moscas (F) e percentagem de parasitismo $(\% \mathrm{P})$ de espécies de parasitóides de diferentes espécies de moscas-das-frutas (Diptera: Tephrtitidae) e espécies frutíferas hospedeiras em diferentes municípios das regiões Norte e Noroeste do Estado do Rio de Janeiro (maio de 2006 a abril de 2007).

\begin{tabular}{|c|c|c|c|c|c|c|c|c|}
\hline Municípios & Fruteiras & $\begin{array}{l}\text { Peso } \\
(\mathrm{g})\end{array}$ & TP & $\mathrm{M}$ & --- & $\begin{array}{l}\text { Moscas-das-frutas } \\
\text { (freqüência relativa) }\end{array}$ & Parasitóides & $\% \mathrm{P}$ \\
\hline \multirow{4}{*}{$\begin{array}{l}\text { Campos dos } \\
\text { Goytacazes }\end{array}$} & Acerola & 482 & 9 & 3 & 4 & $\begin{array}{l}\text { Anastrepha fraterculus } \\
(100 \%)\end{array}$ & - & - \\
\hline & Cajá-mirim & 2390 & 107 & 45 & 25 & A. obliqua (100\%) & Doryctobracon areolatus & 9,4 \\
\hline & Goiaba & 1322 & 26 & 8 & 13 & $\begin{array}{l}\text { A. fraterculus }(85 \%) \text {, } \\
\text { A. sororcula }(15 \%)\end{array}$ & Aganaspis pelleranoi & 11,5 \\
\hline & Maracujá-doce & 1000 & 11 & 3 & 7 & $\begin{array}{l}\text { A. pseudoparallela } \\
(100 \%)\end{array}$ & - & - \\
\hline Itaocara & Goiaba & 3990 & 133 & 11 & 17 & $\begin{array}{l}\text { A. fraterculus }(64 \%) \text {, } \\
\text { A. sororcula }(36 \%)\end{array}$ & - & - \\
\hline \multirow{7}{*}{$\begin{array}{l}\text { São Francisco } \\
\text { do Itabapoana }\end{array}$} & Abiu-amarelo & 3327 & 1709 & 174 & 205 & A. serpentina (100\%) & - & - \\
\hline & Abiu-roxo & 1390 & 23 & 3 & 1 & A. serpentina (100\%) & D. areolatus & 34,8 \\
\hline & Cajá-manga & 1670 & 19 & 10 & 0 & $\begin{array}{l}\text { Anastrepha } \quad \text { spp. } \\
(100 \%)\end{array}$ & D. areolatus & 1,4 \\
\hline & Carambola & 19939 & 1263 & 55 & 50 & $\begin{array}{l}\text { A. obliqua }(56 \%) \text {, } \\
\text { A. sororcula }(38 \%), \text { A. } \\
\text { fraterculus }(4 \%), \text { A. } \\
\text { serpentina }(2 \%)\end{array}$ & D. areolatus & 11,6 \\
\hline & Goiaba & 3070 & 529 & 149 & 165 & $\begin{array}{l}\text { A. fraterculus }(76 \%) \text {, } \\
\text { A. sororcula }(18 \%), \text { A. } \\
\text { zenildae }(6 \%)\end{array}$ & $\begin{array}{l}\text { D. areolatus } \\
\text { A. pelleranoi }\end{array}$ & 1,5 \\
\hline & Pitanga & 210 & 3 & 0 & 3 & $\begin{array}{l}\text { Ceratitis } \quad \text { capitata } \\
(100 \%)\end{array}$ & - & - \\
\hline & Serigüela & 2430 & 223 & 19 & 26 & $\begin{array}{l}\text { A. fraterculus }(62 \%) \text {, } \\
\text { A. sororcula }(38 \%)\end{array}$ & D. areolatus & 18,4 \\
\hline \multirow{2}{*}{$\begin{array}{l}\text { São João da } \\
\text { Barra }\end{array}$} & Araçá-de-coroa & 4200 & 360 & 42 & 66 & $\begin{array}{l}\text { A. sororcula }(74 \%), \text { A. } \\
\text { fraterculus }(22 \%), \text { C. } \\
\text { capitata }(4 \%)\end{array}$ & - & - \\
\hline & Araçá-pera & 1630 & 29 & 4 & 10 & $\begin{array}{l}\text { Anastrepha } \quad \text { spp. } \\
(100 \%)\end{array}$ & - & - \\
\hline Total & & 47050 & 4444 & 526 & 592 & & & \\
\hline
\end{tabular}

goiabeira Paluma, porém, com a predominância de $\boldsymbol{A}$. fraterculus (Tabela 2). Contrariamente, na região Norte de Minas Gerais, a goiaba é principalmente infestada por A. zenildae, ocorrendo com freqüências superiores a $70 \%$, e acredita-se que a predominância dessa espécie seja favorecida pelas condições semi-áridas dessa região (CANAL et al., 1998). No presente estudo, $\boldsymbol{A}$. zenildae só foi obtida de goiabas infestadas por mais uma ou duas espécies de Anastrepha (A. fraterculus e/ou A. sororcula). De acordo com SILVA\& RONCHITELES (2000), é pouco comum duas espécies de moscas-das-frutas infestarem concomitantemente um mesmo fruto, mas esses pesquisadores obtiveram $\boldsymbol{A}$. obliqua e A. antunesi em frutos de Spondias lutea (cajá-mirim ou taberebá). Com base nas listas mais recentes de plantas hospedeiras de moscas-das-frutas no Estado do Rio de Janeiro (AGUIAR-MENEZES \&
MENEZES, 2000; AGUIAR-MENEZES et al., 2004; SOUZA, 2004; SOUZA et al., 2005; SOUZA et al., 2007; SOUZA et al., 2008), a goiaba é registrada pela primeira vez como planta hospedeira de A. zenildae para esse Estado.

Os índices médios de infestação por moscasdas-frutas das goiabas da variedade Paluma foram de 172,3 pupários $\mathrm{kg}^{-1}$ de frutos e 26,5 pupários fruto-1. Esses índices são superiores aos encontrados por RAGA et al. (2006) para a variedade de goiaba Indiana Vermelha (média de 19,4 pupários fruto ${ }^{-1}$, alcançando, no máximo, 49 pupários fruto ${ }^{-1}$ ), a qual foi considerada uma das mais susceptíveis entre outros 10 genótipos de goiaba do banco de germoplasma da Estação de Agricultura Regional APTA. Todavia, na região Norte de Minas Gerais, goiabas da variedade Paluma analisadas por CORSATO (2004) apresentaram um 
índice médio de infestação inferior ao do presente estudo, variando de 25,7 a 28,0 pupários $\mathrm{kg}^{-1}$ de goiabas, dependendo do município de origem das amostras. A partir do número de larvas, CANAL et al. (1998) observaram que os índices de infestação de goiaba variavam de 116 a 2,5 larvas $\mathrm{kg}^{-1}$ (ou 14,8 a 4,4 larvas fruto $^{-1}$ ), em função da época do ano, entre três municípios da região Norte de Minas Gerais. No município de Ferreira Gomes, Estado do Amapá, SILVA \& SILVA (2007) obtiveram os índices de infestação natural da goiaba de 0,24 pupários fruto ${ }^{-1}$ e 5,4 pupários $\mathrm{kg}^{-1}$ de frutos, sendo infestada por cinco espécies de Anastrepha: A. fraterculus, A. obliqua, A. striata, A. antunesi e A. turpiniae, mas a espécie mais abundante foi A. obliqua (76,4\%).

Outros novos registros de planta hospedeira de moscas-das-frutas para Estado do Rio de Janeiro foram obtidos, como carambola para $\boldsymbol{A}$. fraterculus, A. serpentina e A. sororcula, pitanga para C. capitata, acerola para $A$. fraterculus, araçá-de-coroa para $A$. fraterculus, $A$. sororcula e $\boldsymbol{C}$. capitata, abiuamarelo para $A$. serpentina, cajá-manga para $A$. obliqua e serigüela para A. sororcula. Araçá-de-coroa também é registrada pela primeira vez como planta hospedeira de A. sororcula no Brasil, bem como abiu-amarelo e carambola para $\boldsymbol{A}$. serpentina, com base nos dados apresentados por ZUCCHI (2007) e GATTELLI et al. (2008).

Doryctobracon areolatus (Szépligeti) (Hymenoptera: Braconidae) foi a única espécie de parasitóide de larvas obtida de tefritídeos criados em frutos de cajá-mirim coletados em Campos dos Goytacazes e em frutos de carambola, abiu-roxo, cajámanga e serigüela coletados em São Francisco do Itapaboana (Tabela 2). GONÇALVES (1938) observou o parasitismo de A. obliqua (citada como A. fraterculus var. ligata) por D. areolatus (citado como O. cereus) em cajá-mirim, sendo que esta mesma associação parasitóide-hospedeiro foi observada no presente estudo, isto é, A. obliqua parasitada por D. areolatus em cajá-mirim; porém, observou uma taxa de parasitismo mais elevada (56\%) do que a obtida no presente estudo (Tabela 2). Em Seropédica, SOUZA et al. (2007) observaram que D. areolatus foi a principal espécie de parasitóides de larva de moscas-das-frutas infestando cajá-mirim, com um percentual de parasitismo de 58\%, portanto, percentual bem superior ao encontrado no presente estudo (Tabela 2). Resultado semelhante ao do presente estudo foi encontrado por HICKEL (2002), que obteve uma percentagem de parasitismo para larvas de moscas-das-frutas infestando cajá mirim em Viçosa, Minas Gerais, que variou de 2,6\% a 10,7\%.
Em São Francisco do Itabapoana, D. areolatus e Aganaspis pelleranoi (Brèthes) (Hymenoptera: Figitidae) foram obtidos da amostra de $3,07 \mathrm{~kg}$ de goiabas var. Paluma, sendo responsáveis por $1,5 \%$ de parasitismo total das larvas que infestaram esses frutos (Tabela 2). A partir das goiabas individualizadas coletadas nesse município, obtevese um parasitismo total de $1,8 \%$. Em Campos dos Goytacazes, obteve-se apenas A. pelleranoi da amostra de goiaba dessa variedade e o parasitismo total foi de 11,5\% (Tabela 2). Parasitóides não foram obtidos da amostra de goiabas coletada em Itaocara (Tabela 2). GARCIA \& CORSEUIL (2004) e COSTA et al. (2007) também observaram A. pelleranoi como parasitóide de larvas de moscas-das-frutas em goiaba, obtendo $20 \%$ e $10,5 \%$ de parasitismo, respectivamente, portanto, valores superiores ao valor obtido no presente estudo. GARCIA \& CORSEUIL (2004) também observaram um maior número de espécies de parasitóide de larva, incluindo $\boldsymbol{A}$. pelleranoi, porém, não obteve $\boldsymbol{D}$. areolatus. CARVALHO (2005) observou quatro espécies de parasitóides de moscas-das-frutas em goiaba, incluindo D. areolatus e A. pelleranoi, causando 5,08\% de parasitismo.

\section{CONCLUSÕES}

A partir das coletas de moscas-das-frutas realizadas por meio de armadilhas McPhail e de frutos infestados, pode-se concluir que, nas regiões Norte e Noroeste fluminense, ocorrem 16 espécies de moscasdas-frutas da família Tephritidae, sendo a maioria do gênero Anastrepha; porém, apenas três espécies ( $A$. fraterculus, A. sororcula e A. zenildae) infestam goiabas da variedade comercial Paluma. Outras fruteiras das famílias Anacardiaceae, Malpighiaceae, Myrtaceae, Oxalidaceae, Passifloraceae e Sapotaceae são plantas hospedeiras de moscas-das-frutas, garantindo a sobrevivência das mesmas na entressafra da goiabeira, funcionando como hospedeiros alternativos. Além disso, apenas Doryctobracon areolatus e Aganaspis pelleranoi parasitam larvas de moscas-das-frutas em frutos de goiabeira da variedade Paluma e de outras fruteiras nessas regiões.

\section{AGRADECIMENTOS}

À Coordenação de Aperfeiçoamento de Pessoal de Nível Superior (CAPES), pela concessão de bolsa de mestrado ao primeiro autor. À Fundação Carlos Chagas Filho de Amparo à Pesquisa do Estado do Rio de Janeiro (FAPERJ), pela concessão auxílio à pesquisa (modalidade APQ1 - processo no E-26/ 170.941/2003). 


\section{REFERÊNCIAS}

AGUIAR-MENEZES, E.L.; MENEZES, E.B. Rio de Janeiro. In: MALAVASI, A.; ZUCCHI, R.A. (Eds.). Moscas-das-frutas de importância econômica no Brasil: conhecimento básico e aplicado. Ribeirão Preto: FAPESP-Holos, 2000. p.259-263.

AGUIAR-MENEZES, E.L. et al. Diversity of fly species (Diptera: Tephritoidea) from Passiflora spp. and their hymenopterous parasitoids in two municipalities of the Southeastern Brazil. Neotropical Entomology, Londrina, v.33, n.1, p.113-116, 2004.

BRANDÃO, A.S.P. O pólo de fruticultura irrigada no norte e noroeste fluminense. Política Agrícola, Brasília, v.13, n.2, p.78-86, 2004

CANAL, N.A.D. et al. Níveis de infestação de goiaba por Anastrepha zenildae Zucchi (Diptera: Tephritidae), em pomares comerciais do norte de Minas Gerais. Anais da Sociedade Entomológica do Brasil, Londrina, v.27, p.657-661, 1998.

CANAL, N.A.D.; ZUCCHI, R.A. Parasitóides - Braconidae. In: MALAVASI, A.; ZUCCHI, R.A. (Eds.). Moscas-das-frutas de importância econômica no Brasil: conhecimento básico e aplicado. Ribeirão Preto: FAPESP-Holos, 2000. p.119-126.

CARVALHO, R.S. Avaliação das liberações inoculativas do parasitóide exótico Diachasmimorpha longicaudata (Ashmead) (Hymenoptera: Braconidae) em pomar diversificado em Conceição de Almeida, BA. Neotropical Entomology, Londrina, v.34, n.5, p.799-805, 2005.

CORSATO, C. D.A. Moscas-das-frutas (Diptera: Tephritidae) em pomares de goiaba no norte de Minas Gerais: biodiversidade, parasitóides e controle biológico. 2004. 95f. Tese (Doutorado em Entomologia) - ESALQ, USP, Piracicaba, SP.

COSTA, R.I.F. et al. Parasitismo em Anastrepha sp. (Diptera: Tephritidae) por Aganaspis pelleranoi (Brèthes, 1924) e Dicerataspis sp. (Hymenoptera: Figitidae: Eucoilinae). Ciência e Agrotecnologia, Lavras, v.31, n.3, p.720-723, 2007.

FERRARA, F.A.A. et al. Novos registros de moscas-das-frutas (Diptera: Tephritidae) no estado do Rio de Janeiro. Neotropical Entomology, Londrina, v.33, n.6, p.797-798, 2004.

FOOTE, R.H. Fruit fly genera in the south of the United States. Washington: USDA, 1980. 79p. (U.S.D.A., Science and Education Administration, Technical Bulletin 1600).

GARCIA, F.R.M.; CORSEUIL, E. Native hymenopteran parasitoids associated with fruit flies (Diptera: Tephritidae) in Santa Catarina state, Brazil. Florida Entomologist, Lutz, v.87, n.4, p.517-521, 2004.

GATTELLI, T. et al. Moscas frugívoras associadas a mirtáceas e laranjeira "Céu" na região do vale do Rio Caí, Rio Grande do Sul, Brasil. Ciência Rural, Santa Maria, v.38, n.1, p.236239, 2008. Disponível em: http://www.scielo.br/ scielo.php? script=sci_arttext\&pid=S0103-
$84782008000100038 \& \operatorname{lng}=$ pt\&nrm=iso\&tlng=pt. $\quad$ Doi: 10.1590/S0103-84782008000100038.

GONÇALVES, C.R. As moscas das frutas e seu combate. Rio de Janeiro: Ministério da Agricultura, Departamento Nacional de Produção Vegetal, Serviço de Defesa Sanitária Vegetal, 1938. 48p.

GOULD, W.P.; RAGA, A. Pests of guava. In: PEÑA, J.E. et al. (Eds.). Tropical fruit pests and pollinators: biology, economic importance, natural enemies and control. Wallingford: CABI, 2002. p.295-313.

GUIMARÃES, J.A. et al. Parasitóides - Figitidae (Eucoilinae). In: MALAVASI, A.; ZUCCHI, R.A. (Eds.). Moscas-das-frutas de importância econômica no Brasil: conhecimento básico e aplicado. Ribeirão Preto: FAPESP-Holos, 2000. p.127-135.

HICKEL, E.R. Espessura da polpa como condicionante do parasitismo de moscas-das-frutas (Diptera: Tephritidae) por Hymenoptera: Braconidae. Ciência Rural, Santa Maria, v.32, n.6, p.1005-1009, 2002. Disponível em: http://www.scielo.br/ scielo.php? script=sci_arttext\&pid=S 0103 $84782002000600014 \& \operatorname{lng}=$ en\&nrm=iso\&tlng=pt. Doi: $10.1590 /$ S0103-84782002000600014.

RAGA, A. et al. Susceptibility of guava genotypes to natural infestation by Anastrepha spp. (Diptera: Tephritidae) in the municipality of Monte Alegre do Sul, state of São Paulo, Brazil. Neotropical Entomology, Londrina, v.35, n.1, p.121-125, 2006.

SEAAPI. Secretaria de Agricultura, Abastecimento, Pesca e Desenvolvimento do Interior do Estado do Rio de Janeiro. Programas setoriais, frutificar. Capturado em 12 jun. 2008. Online. Disponível na Internet: http://www.seaapi.rj.gov.br/ setoriais.asp.

SILVA, W.R.; SILVA, R.A. Levantamento de moscas-das-frutas e de seus parasitóides no município de Ferreira Gomes, Estado do Amapá. Ciência Rural, Santa Maria, v.37, n.1, p. 265 268, 2007. Disponível em: http://www.scielo.br/ scielo.php?script=sci_arttext\&pid=S 0103 . $84782007000100043 \& \operatorname{lng}=$ pt $\&$ nrm $=$ iso \& $\operatorname{lng}=$ pt. Doi: 10.1590/S0103-84782007000100043.

SILVA, N.M.; RONCHI-TELES, B. Amapá, Amazonas, Pará, Rondônia e Roraima. In: MALAVASI, A.; ZUCCHI, R.A. (Eds.) Moscas-das-frutas de importância econômica do Brasil: conhecimento básico e aplicado. Ribeirão Preto: FAPESPHolos, 2000. p.203-209.

SOUZA, J.F. Aspectos ecológicos das populações de moscas frugívoras (Diptera: Tephritoidea) no município de Araruama, Estado do Rio de Janeiro. 2004. 78f. Dissertação (Mestrado em Fitotecnia) - UFRRJ, Seropédica, RJ.

SOUZA, J.F. et al. Diversidade de moscas-das-frutas em pomares de citros no município de Araruama, RJ. Ciência Rural, Santa Maria, v.38, n.2, p.518-521, 2008. Disponível em: http:// www.scielo.br/scielo.php?script=sci_arttext\&pid=S0103$84782008000200035 \& \operatorname{lng}=$ pt\&nrm=iso\&tlng=pt. Doi: $10.1590 /$ S0103-84782008000200035. 
SOUZA, S.A.A. et al. Infestação natural de moscas frugívoras (Diptera: Tephritoidea) em café arábica, sob cultivo orgânico arborizado e a pleno sol, em Valença, RJ. Neotropical Entomology, Londrina, v.34, n.4, p.639-648, 2005.

SOUZA, S.A.A. et al. Índices de infestação de Spondias lutea L. por moscas-das-frutas (Diptera: Tephritdae) e seus parasitóides no município de Seropédica, RJ. Magistra, Cruz das Almas, v.29, n.1, p.25-30, 2007.

ZUCCHI, R.A. Diversidad, distribución y hospederos del género Anastrepha en Brasil. In: ORTIZ-HERNANDÉZ, V. (Ed.). Moscas de la fruta en Latinoamérica (Diptera:
Tephritidae): diversidad, biología y manejo. Distrito Federal, México: S y G Editores, 2007. p.77-100.

ZUCCHI, R.A. Mosca-do-mediterrâneo, Ceratitis capitata (Diptera: Tephritidae). In: VILELA, E.F. et al. (Eds.). Histórico e impacto das pragas introduzidas no Brasil. Ribeirão Preto: Holos, 2001. p.15-22.

ZUCCHI, R.A. Taxonomia. In: MALAVASI, A.; ZUCCHI, R.A. (Eds.). Moscas-das-frutas de importância econômica no Brasil: conhecimento básico e aplicado. Ribeirão Preto: FAPESP-Holos, 2000. p.13-24. 